\title{
Applicability Analysis of Aggregated Crash Index (ACI) in Freeways
}

\author{
Yan Kuang ${ }^{1}$ and Xiaobo $\mathbf{Q u}{ }^{2}$
}

\begin{abstract}
Traffic safety especially freeway safety has become of increasing concern of transport agencies due to the increasing losses to society. The crash surrogate, which is defined as the measure of crash proximity, is widely accepted as an alternative to crash data by taking advantage of many merits. With the increased travel demand, freeways frequently operate at capacity or even in a state of synchronized flow with high speed and small headway. Under this kind of state, even a slight disturbance might result in a rear-end crash or even a chain collision (multi-vehicle collision), which could consequently lead to serious health, economic and environmental problems. However, the traditional surrogate measures could not perform very well in this state due to some limitations. Surrogate measure Aggregated Crash Index (ACI), a tree-structure based indicator, is designed based on a hypothetical disturbance imposed. With the hypothetic disturbance, ACI can be applied to any car following scenario without any restricted conditions. In this paper, we carry out a case study to illustrate how ACI is applied to the distinct car-following scenarios. The result shows that ACI has more comprehensive capability and applicability on representing crash risk.
\end{abstract}

Keywords: proactive approach, reactive approach, surrogate measure.

\section{Introduction}

As the leading cause of death for young people aged 15 - 29 years, the motor-vehicle crash has been recognized as a major health problem. In Australia, the social cost of vehicle crashes has been estimated as a devastating AUD 27 billion per annum which is around 2\% of the GDP (DIRD, 2013). Due to the high speeds, the crashes on freeways are found having higher severity than those on urban streets. It is reported that the proportion of fatal crashes on rural freeways is significantly higher than that on urban roads and streets (US DOT, 2005). Freeways often operate at capacity or even in a state of saturated flow under high travel demand, with similarly high speeds and small headways. During this kind of state, a rearend crash or even a chain collision (multi-vehicle collision) can be caused with a high probability by even a slight disturbance might. Due to the serious health, economic and environmental problems caused by crashes, it is of great importance to improve traffic safety.

In order to reduce the number of crashes and enormous losses to society, researchers have been seeking solutions to improve traffic safety for decades (Lord and Mannering, 2010; Savolainen et al., 2011; Chiou and Fu, 2013; Qu and Meng, 2014). Surrogate measure, which is a measure of traffic conflicts, has been widely proposed and utilized into traffic safety for

\footnotetext{
$1 \quad$ PhD candidate, School of Engineering, Gold Coast Campus, Griffith University, Queensland, 4222, Australia. Email: y.kuang@griffith.edu.au.

2 Senior Lecturer, School of Engineering, Gold Coast Campus, Griffith University, Queensland, 4222, Australia. Email: x.qu@griffith.edu.au.
} 
decades (e.g. Chin and Quek, 1997; Gettman and Head, 2007; Tarko, 2012; Xu et al., 2012). By taking advantage of many merits, crash surrogate measure has been well recognized as a good safety indicator on predicting crashes (Tarko et al., 2009). At first, traffic conflicts occur much more frequently than crashes, with strong statistical and causal relationships to crashes. Secondly, by representing the state between safety and a crash, traffic conflicts can reflect the potential crash causality and crash mechanism. Last but not least, surrogate measure is a proactive approach which can assess safety before a crash occurs. Accordingly, surrogate measures can provide better performance in terms of assessing safety and predicting crashes than the historical crash data, due to their more frequent occurrence and strong causal relationship with crashes (Minderhoud and Bovy, 2001; Wu and Jovanis, 2012\&2013).

By taking advantage of these merits, various surrogate measures have been proposed and designed to measure traffic conflicts. The surrogate indicator Time To Collision (TTC) which is proposed by Hayward (1972), is represented as the time remains until a collision between two vehicles would have occurred if both vehicles keep speed unchanged during the whole process. As one of the most well-recognized microscopic safety indicators, TTC has been widely applied to evaluate the level of safety in different situations of traffic (Vogel, 2003; Meng and Qu, 2012; Sayed et al., 2013; Qu et al., 2014a\&b; Qu et al., 2015). Cooper and Ferguson (1976) proposed Deceleration Rate to Avoid a Crash (DRAC) as the minimum deceleration rate required by the following vehicle to avoid a crash if the leading vehicle keeps its speed unchanged during the whole process. Based on DRAC, another surrogate measure Crash Potential Index (CPI) is proposed by Cunto and Saccomanno (2008) as the probability that a given vehicle's DRAC exceeds its Maximum Available Deceleration Rate (MADR) or braking capacity. The surrogate indicator CPI is broadly used to evaluate the road risk in safety analysis (Cunto et al., 2009; Meng and Weng, 2011; Guido et al., 2011). The surrogate Proportion of Stopping Distance (PSD) is defined by Allen et al. (1978) as the ratio between the remaining distance and its minimum acceptable stopping distance which is based on the assumption of maximum deceleration rate used. PSD is also used for safety evaluation and regarded as a good surrogate indicator widely (Guido et al., 2011). The previous researches suggest that these surrogate measures perform well in various situations, such as intersections, expressways, tunnels etc. However, they have been found unsuitable for measuring conflicts on freeways, especially saturated freeways where a minor disturbance might cause a rear-end crash or even a chain collision due to high speeds and small headways (Kuang et al., 2015). Potential risks cannot be measured by these surrogate measures in such situations because of the boundary conditions on speed. In other words, these measures are only suitable for scenarios where the speed of the following vehicle is greater than that of the leading vehicle (Kuang et al., 2015). Consequently, the potential risks on freeways cannot be accurately measured by them.

Due to the unsuitability of the current surrogate measures for freeways, a new surrogate Aggregated Crash Index (ACI) is proposed by Kuang et al. (2015) to better measure the conflicts on freeways in a saturated state. By taking into account more interactions without any boundary conditions, ACI can better measure the conflicts on freeways in a saturated state with all car-following scenarios examined. This paper aims to examine the applicability of this novel surrogate indicator by using traffic data provided by the Federal Highway Administration (FHWA) of the United States Department of Transportation (US DOT). We use a case study to examine the applicability of ACI and other traditional surrogate Time to Collision (TTC), Crash Potential Index (CPI) and Proportion of Stopping Distance (PSD), with 96,500 randomly extracted discrete Instantaneous Car-following Scenarios. The result 
shows that the surrogate measure ACI has wider applicability than other surrogates. For those car-following scenarios in which the speed differences are not apparent, ACI can evaluate the detailed risks while the other surrogate measures cannot.

\section{Methodology}

Kuang et al. (2015) modified Davis’ probabilistic causal model (Davis et al., 2011) by simply imposing a hypothetical disturbance on the leading vehicle. Figure 1 shows the crash mechanism where the evasive action is determined and crash outcome is identified. In this modified probabilistic model, the evasive action will be taken by the following vehicle, based on the disturbance imposed on the leading vehicle and the initial conditions. Further, by examining the evasive action, the crash outcome can be identified. As a block diagram, the hypothetical disturbance and the crash outcome is regarded as the input and output respectively, while the crash mechanism can be deemed as the process in this model.

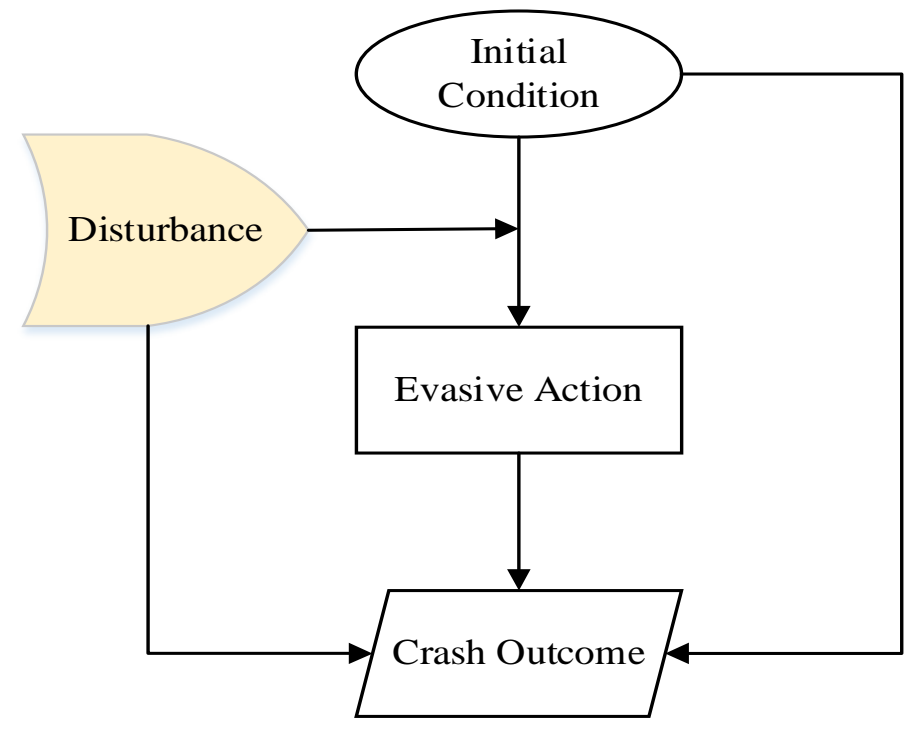

Figure 1. Modified probabilistic causal model (Kuang et al., 2015)

Based on the proposed probabilistic causal model, Kuang et al. (2015) further developed a tree structure to describe total eight leaf nodes with different crash outcomes. By analysing the probability and crash outcome of each leaf node, the crash risk of the entire car-following scenario can be obtained. For any car-following scenario, there will be 8 possible conflict types which are defined four levels of conditions which are shown in Table 1. Kuang et al. (2015) concluded that the probability of each car-following scenario relies on the four levels of conditions which is shown as

$$
\begin{aligned}
P\left(L_{j}\right)= & P\left(\tau_{L_{j}}^{4} \mid \tau_{L_{j}}^{1}, \tau_{L_{j}}^{2}, \tau_{L_{j}}^{3}\right) \cdot P\left(\tau_{L_{j}}^{3} \mid \tau_{L_{j}}^{1}, \tau_{L_{j}}^{2}\right) \cdot P\left(\tau_{L_{j}}^{2} \mid \tau_{L_{j}}^{1}\right) \cdot P\left(\tau_{L_{j}}^{1}\right) \cdot \delta_{j} \\
& +P\left(\tau_{L_{j}}^{2} \mid \tau_{L_{j}}^{1}\right) \cdot P\left(\tau_{L_{j}}^{1}\right) \cdot\left(1-\delta_{j}\right) \\
& \forall \delta_{j}= \begin{cases}1 & j \in\{3,4,5,6,7,8\} \\
0 & j \in\{1,2\}\end{cases}
\end{aligned}
$$


where $\tau_{L_{j}}^{n}$ is the $n^{\text {th }}$ level of condition for leaf node $j, \delta_{j}$ is a switch variable with value 1 or 0 in different situations. In order to aggregate all the existing crash probabilities of 8 possible conflicts, a new surrogate measure ACI is proposed by Kuang et al. (2015) to represent the accumulation of the crash probabilities of all possible conflicts. Based on the notion of the surrogate measure ACI, the overall crash risk for any car-following scenario can be represented as

$$
A C I=\sum_{j=1}^{8} C R_{L_{j}}=\sum_{j=1}^{8} P\left(L_{j}\right) \cdot C_{L_{j}}
$$

where $C_{L_{j}}$ is the cost incurred at each leaf node $L_{j}$. ACI thus directly represents the crash risk, by considering additional factors such as reaction time and braking capacity. For any car-following scenario $i$, the ACI over the time period $T$ can be represented as

$$
A C I_{i}=\frac{\sum_{t=0}^{N} A C I_{i}(t) \cdot \Delta t}{T}
$$

where $A C I_{i}(t)$ denotes the ACI value for the $i^{\text {th }}$ car-following scenario at discrete time $t$; $N$ and $\Delta t$ are the total number and duration of the time intervals; $T$ is the total time duration investigated, where $T=N \cdot \Delta t$. In this paper, as suggested by Triggs and Harris (1982), the divers' reaction time is this paper follows a lognormal distribution with a mean of 0.92 second and a standard deviation of 0.28 second. The MADR is represented by truncated normal distributions (AASHTO, 2004; Cunto and Saccomanno, 2008).

\section{Applicability analysis}

In order to apply the proposed methodology, 96,500 discrete Instantaneous Car-following Scenarios (ICSs) are extracted randomly from the trajectory data of the I-80 provided by FHWA, each with time duration 1/15 second. The database has been widely used in microscopic traffic analysis (e.g. Qu et al., 2015b). In this research, all 96,500 ICSs are evaluated by ACI and the traditional surrogate measures of CPI, TTC and PSD respectively. In order to represent the risk for each scenario, the concept of individual risk is introduced. In this study, the individual risk $\left(I R_{i}\right)$ is defined as the likelihood of a collision occurring to individual traveller $i$. For each ICS, the individual risk can be expressed as the difference between the surrogate value and its threshold, or mathematically,

$$
I R_{i j}= \begin{cases}S_{j}^{*}-S_{i j} & \text { if } S_{j}^{*}>S_{i j} \\ 0 & \text { otherwise }\end{cases}
$$

where $I R_{i j}$ represents the individual risk of discrete ICS $i$ measured by surrogate $j$; $S_{i j}$ denotes the surrogate value for discrete ICS $i$ measured by surrogate $j ; S_{j}^{*}$ is the threshold of surrogate measure $j$. Since the surrogate measures ACI and CPI represent risk, the individual risk of an ICS can be denoted by these two surrogate measures directly. The thresholds for TTC and PSD are set to 4 seconds and 1 respectively (Meng and Qu, 2012; Guido et. al., 2011). Using the data from the I80, the speeds, headway and vehicle lengths for each car-following scenario are all obtainable. In order to investigate the change in the risk over time, we take just the 1,500 ICSs with the highest risk. Based on the concept of individual risk, these 1,500 ICSs are evaluated by the surrogate measures ACI, CPI, TTC and PSD respectively according to their notions. As can be seen in Figure 2, the individual risk of these 1,500 ICSs gradually decreases in the four pictures. However, some ICSs are 
found to have high risk according to the ACI but zero risk according to the surrogate measures CPI, TTC and PSD.
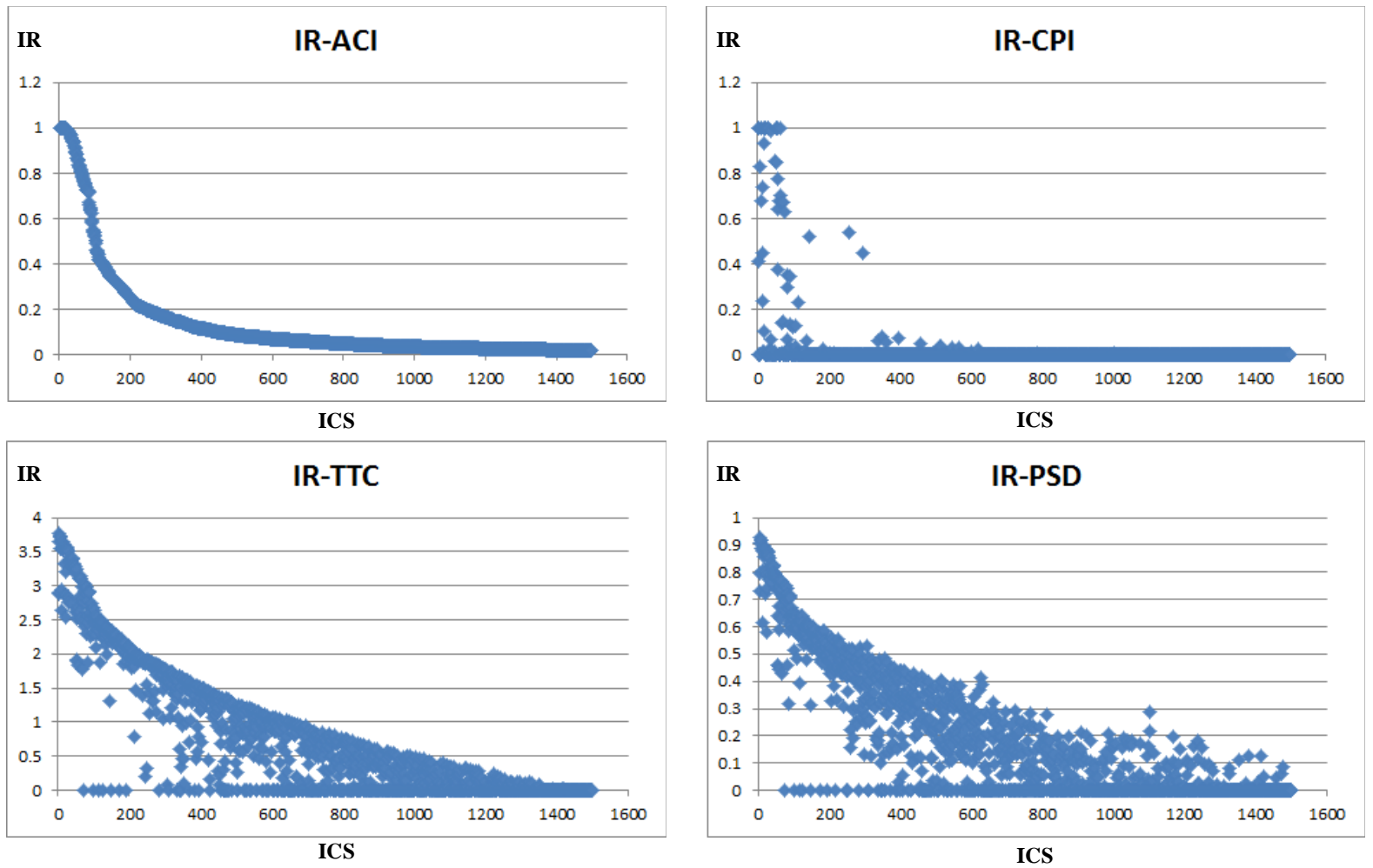

Figure 2. Risk analysis for all scenarios

In order to investigate the risk in a car-following scenario with an obvious speed difference, we choose one scenario in which the speed of the leading vehicle is much less than that of the following vehicle, due to lane changing by some vehicle up ahead. The total time period investigated for this car-following scenario covers 185 time frames. Using the same four surrogate measures, the individual risk of this car-following scenario is estimated. As shown in Figure 3, the individual risk is found to be high at first and then to decrease gradually according to TTC, ACI and PSD, while it increases at first and then decreases according to the CPI. In general, ACI has a similar risk trend over time to TTC and PSD. 

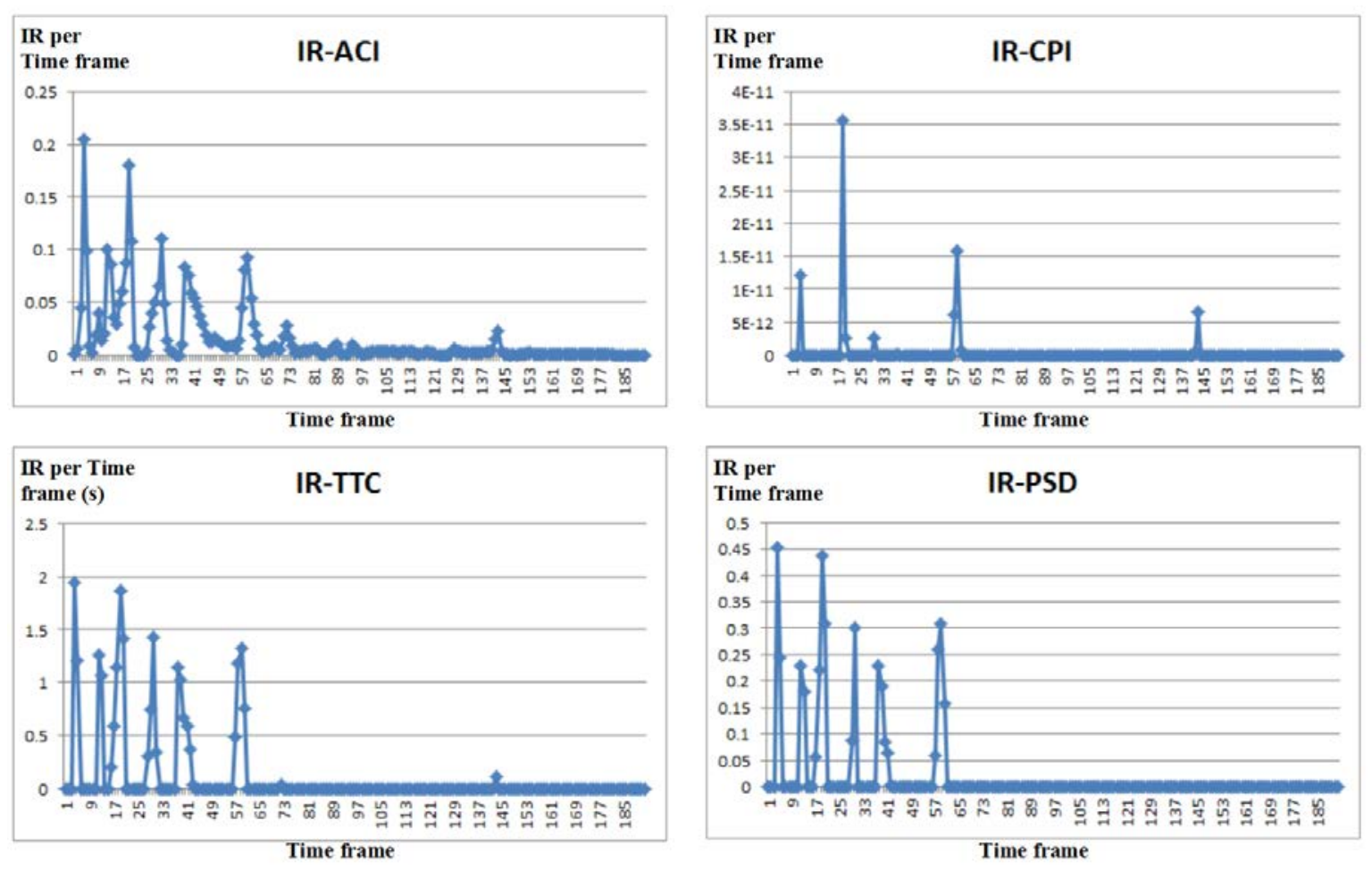

Figure 3. Risk analysis for car-following scenario \#1

We further check one scenario in which the speed of the following vehicle is consistently less than or equal to that of the leading vehicle. A total of 451 time frames are studied. The average time headway for this scenario is 0.61 seconds, which is very risky in a freeway situation. Figure 4 shows the risk in this car-following scenario according to the four surrogate measures. The individual risk is suggested to be zero by CPI, TTC and PSD, while ACI shows it to vary over time. In this car-following scenario, the vehicles drive with similar speeds and a small time gap. However, the risk cannot be identified by CPI, TTC and PSD. It should be pointed out that this scenario is also associated with high speeds and low headways for both vehicles. ACI identifies this scenario as risky, while the other surrogate measures identify it as safe. In other words, the other surrogate measures are only applicable to car-following scenarios in which the following vehicle is moving faster than the leading vehicle. In contrast, the ACI can be used under any car-following conditions. 

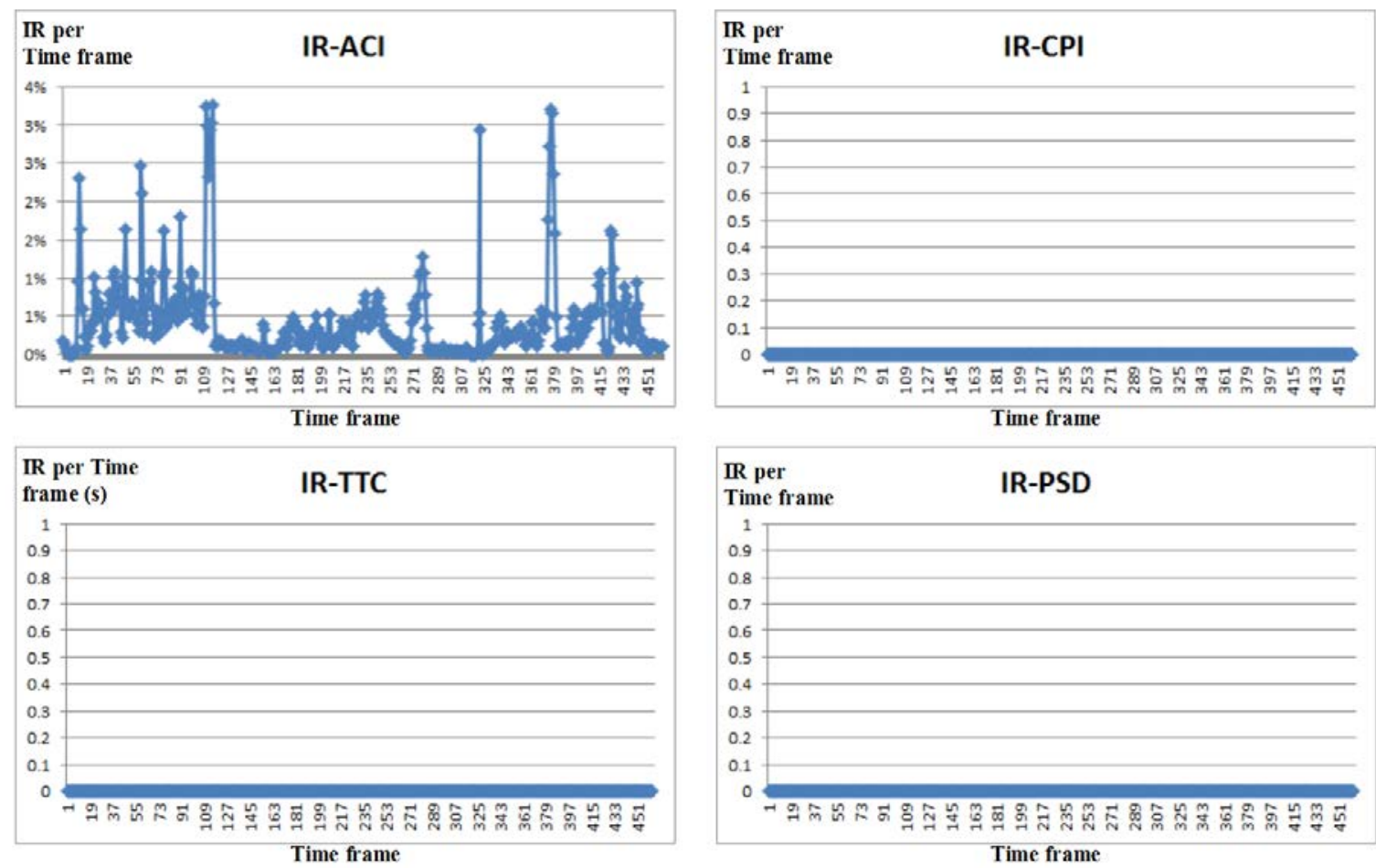

Figure 4. Risk analysis for car-following scenario \#2

\section{Findings and discussions}

The results show the following: (1) The ACI depicts a similar time trend to traditional surrogate measures (CPI, TTC and PSD) for car-following scenarios in which the speed of the following vehicle is obviously greater than that of the leading vehicle. (2) For scenarios in which the speed of the following vehicle is not apparently greater than that of the leading one, the ACI produces different results to the traditional surrogate measures. This is due to the definition of the traditional surrogate measures. According to the notions of CPI, TTC and PSD, all scenarios in which the speed of the following vehicle is not greater than that of the leading vehicle are regarded as safe. That is to say, the condition used to determine the risk is the speed differential. Therefore, it is impossible to identify the risks in other scenarios using the traditional surrogate measures. As discussed in the methodology section, the ACI is proposed based on a hypothetical disturbance and is derived from the crash mechanism; it can be used in any car-following scenario, even when the leading vehicle's speed is greater than that of the following vehicle. Furthermore, the ACI takes into account more important variables, namely the reaction time and the MADR, better representing the risk by taking into account the complex crash mechanism. Through these merits, the surrogate measure ACI is able to represent the risk more accurately and has wider applicability. 


\section{Conclusion}

This paper proposes a novel methodology for producing a surrogate measure based on a hypothetical disturbance. Under this hypothetical disturbance to the leading vehicle, the following vehicle's action taken to avoid a crash is based on a probabilistic causal model. Then a tree structure is developed to describe the eight possible conflict types under the model. The surrogate measures of BRAD and ACI are proposed to measure the minimum braking rate required and the aggregated crash risk respectively. With a hypothetical disturbance, there is no boundary conditions, that is to say, all car-following scenarios can be evaluated by the ACI. By taking into account the reaction time and the MADR, the ACI provides more accurate results. The example we provide shows that the ACI can evaluate car-following scenarios in which the speed differences are not apparent, while the other surrogate measures cannot. Therefore, the ACI provides more comprehensive representations of the risk than the traditional surrogate measures. It would be of great interest to conduct further applications in freeway safety.

\section{References}

AASHTO, 2004. Policy on Geometric Design of Highways and Streets. American Association of State Highway and Transportation Officials. Washington, DC.

Allen, B. L., Shin, B. T., Cooper, D. J., 1978. Analysis of traffic conflicts and collision. Transportation Research Record 667, 67-74.

Chin, H.C., Quek, S.T., 1997. Measurement of traffic conflicts. Safety Science 26,169-185.

Chiou, Y.C. and Fu, C., 2013. Modelling crash frequency and severity using the multinomial-generalized Poisson model. Accident Analysis and Prevention, 50, 73-82.

Cooper, F., Ferguson, N., 1976. Traffic studies at t-junctions - a conflict simulation model. Traffic Engineering and Control 17, 306-309.

Cunto, F., and Saccomanno F.F., 2008. Calibration and validation of simulated vehicle safety performance at signalized intersections. Accident Analysis and Prevention. 40, 11711179.

Cunto, F., Duong, D., Saccomanno, F., 2009. Comparision of Simulated Freeway Safety Performance with Observed Crashes. Transport. Research Record 2103, 88-97.

Davis, G., Hourdos, J., Xiong, H., Chatterjee, I., 2011. Outline of a Causal model of Traffic Conflicts and Crashes. Accident Analysis and Prevention 43, 1907-1979.

Department of Infrastructure and Regional Development (DIRD), 2013. Road safety. http://www.infrastructure.gov.au/roads/safety.

Gettman, D., Head, L., 2007. Surrogate safety measures from traffic simulation models. Transportation Research Record 1840, 104-115.

Guido, G., Saccomanno, F., Vitale, A., Astarita, V., Festa, D., 2011. Comparing safety performance measures obtained from video capture data. Journal of Transportation Engineering 137(7), 481-491.

Hayward, J.C., 1972. Near miss determination through use of a scale of danger. Transportation Research Record 384, 24-34.

Kuang, Y., Qu, X., Wang, S., 2014. Propagation and dissipation of crash risk on saturated freeways. Transportmetrica B: Transport Dynamics, 2(3), 203-214.

Kuang, Y., Qu, X., Wang, S., 2015. A Tree-structured Crash Surrogate Measure for Freeways. Accident Analysis and Prevention 77, 137-148. DOI:10.1016/j.aap.2015.02.007. 
Lord, D., and Mannering, F.L., 2010. The statistical analysis of crash frequency data: a review and assessment of methodological alternatives. Transportation Research Part A, 44(5), 291-305.

Meng, Q., Qu, X., 2012. Estimation of rear-end vehicle crash frequencies in urban road tunnels. Accident Analysis and Prevention 48, 254-263.

Meng, Q., Weng, J., 2011. Evaluation of rear-end crash risk at work zone using work zone traffic data. Accident Analysis and Prevention 43, 1291-1300.

Minderhoud, M., Bovy, P., 2001. Extended time-to-collision measures for road traffic safety assessment. Accident Analysis and Prevention 33, 89-97.

Qu, X., Kuang, Y., Oh, E., Jin, S., 2014a. Safety Evaluation for Expressways: A Comparative Study for Macroscopic and Microscopic Indicators. Traffic Injury Prevention 15(1), 89-93.

Qu, X., Yang, Y., Liu, Z., Jin, S., and Weng, J., 2014b. Potential crash risks of expressway on-ramps and off-ramps: a case study in Beijing, China. Safety Science, 70, 58-62.

Qu, X., and Meng, Q., 2014. A note on hotspot identification for urban expressways. Safety Science, 66, 87-91.

Qu, X., Jin, S., and Weng, J., 2015a. Analysis of the Relationship between Aggregated Traffic Volume and Traffic Conflicts on Expressways. Transportmetrica A: Transport Science, DOI:10.1080/23249935.2015.1059378

Qu, X., Wang, S., and Zhang, J., 2015b. On the fundamental diagram for freeway traffic: A novel calibration approach for single-regime models. Transportation Research Part B, 73, 91-102.

Savolainen, P.T., Mannering, F.L., Lord, D., Quddus, M.A., 2011. The statistical analysis of highway crash-injury severities: A review and assessment of methodological alternatives. Accident Analysis \& Prevention 43(5), 1666-1676.

Sayed, T., Zaki, M.H., Autey, J., 2013. Automated safety diagnosis of vehicle-bicycle interactions using computer vision analysis. Safety Science 59, 163-172.

Tarko, A. P., 2012. Use of crash surrogates and exceedance statistics to estimate road safety. Accident Analysis and Prevention 45, 230-240.

Tarko, A., Davis, G., Saunier, N., Sayed, T., Washington, S., 2009. White Paper: Surrogate Measures of Safety. Committee on Safety Data Evaluation and Analysis (ANB20).

Triggs, T., Harris, W., 1982. Reaction Time of Drivers to Road Stimuli. Technical Report, Monash University, Human Factor Group, Department of Psychology.

United States Department of Transportation (US DOT), 2005. Traffic safety fact 2005. NHTSA's national center for statistic \& analysi (NCSA). http://wwwnrd.nhtsa.dot.gov/Pubs/810631.pdf.

Wu, K.F., Jovanis, P.P., 2012. Crashes and crash-surrogate events: Explanatory modelling with naturalistic driving data. Accident Analysis and Prevention 45, 507-516.

Wu, K.F., Jovanis, P.P., 2013). Defining, screening, and validating crash surrogate events using naturalistic driving data. Accident Analysis and Prevention 61, 10-22.

Xu, C., Liu, P., Wang, W., Li, Z., 2012. Evaluation of the impacts of traffic states on crash risks on freeways. Accident Analysis and Prevention 47, 162-171. 